\title{
Impact of Thorium Capture Cross Section Uncertainty on the Thorium Utilized ADS Reactivity Calculation
}

\author{
Thanh Mai Vu ${ }^{1,2}$ and Takanori Kitada ${ }^{1}$ \\ ${ }^{1}$ Osaka University, 2-1, Yamadaoka, Suita-shi, Osaka 565-0871, Japan \\ ${ }^{2}$ Hanoi University of Science, 334 Nguyen Trai, Thanh Xuan, Hanoi, Vietnam \\ Correspondence should be addressed to Thanh Mai Vu; m-vu@ne.see.eng.osaka-u.ac.jp
}

Received 15 June 2014; Accepted 3 August 2014; Published 17 August 2014

Academic Editor: Eugenijus Ušpuras

Copyright (C) 2014 T. M. Vu and T. Kitada. This is an open access article distributed under the Creative Commons Attribution License, which permits unrestricted use, distribution, and reproduction in any medium, provided the original work is properly cited.

Recently, the researches on fast neutron spectrum system utilized thorium fuel are widely conducted. However, the recent thorium cross section libraries are limited compared to uranium cross section libraries. The impact of thorium cross section uncertainty on thorium fuel utilized accelerator driven system (ADS) reactivity calculation is estimated in this study. The uncertainty of the $k_{\text {eff }}$ caused by ${ }^{232}$ Th capture cross section of JENDL- 4.0 is about $1.3 \%$. The uncertainty of JENDL- 4.0 is needed to be enhanced to provide more reliable results on reactivity calculation for the fast system. The impact of uncertainty of ${ }^{232} \mathrm{Th}$ capture cross section of ENDF/B-VII is small (0.1\%). Therefore, it will cause no significant impact of the thorium cross section library on the thorium utilized ADS design calculation.

\section{Introduction}

Thorium with its abundance in nature attracts a considerable attention to be a replacing fuel for uranium fuel. Moreover, with the feature that the TRUs production during an operating cycle is drastically reduced compared with the use of uranium, fuel cycle is favorable for the TRUs eliminating system such as the energy amplifier demonstrator facility (EADF) [1], thorium-fueled fast breed reactors (FBRs) [2], or the seed and blanket thorium-reprocessed fuel ADS [3]. However, experimental data and research results of thorium fuel are very limited compared with uranium fuel data and the calculation results of the thorium fuel utilized system could not be evaluated properly. In the previous publication, in order to verify the accuracy of ${ }^{232} \mathrm{Th}$ cross section library in the thermal neutron spectrum system, the experiment on thorium critical cores was conducted. By comparing the experimental and calculated results, the underestimation of ${ }^{232} \mathrm{Th}$ capture cross section of JENDL-4.0 [4] was found to cause the overestimation of effective multiplication factor of the thermal system, about $0.78 \%$ [5]. As mentioned above, thorium is widely utilized as the fuel for fast reactor concepts, especially ADS. Thus, it is necessary to investigate the impact of thorium capture cross section uncertainty on the reactivity calculation for the fast system utilized thorium fuel. In order to do so, the sensitivity calculations of effective multiplication factor of the seed and blanket thorium-reprocessed fuel ADS for ${ }^{232} \mathrm{Th}$ capture cross section of JENDL-4.0 and ENDF/BVII library were done. The uncertainty of the reactivity calculation results for ${ }^{232} \mathrm{Th}$ capture cross section of these two cross section libraries is estimated and presented in this paper.

\section{Calculation Model}

The calculation model employed in this study is the $2 \mathrm{D}$ seed and blanket fuel ADS model [3]. The seed and blanket thorium-reprocessed fuel ADS was designed in order to achieve the efficient transuranic elements (TRUs) transmutation, simplification in the assembly fabrication, and in-core management and Th fuel utilization to produce energy. Leadbismuth target is placed at center of the core. Sodium is used as coolant of the ADS. The hexagonal assembly is used in the subcritical core. 84 assemblies of individual reprocessed fuel in oxide form are inserted as seed and 96 assemblies of thorium in the oxide form are inserted as blanket of the core to make a heterogeneous configuration. The utilization 


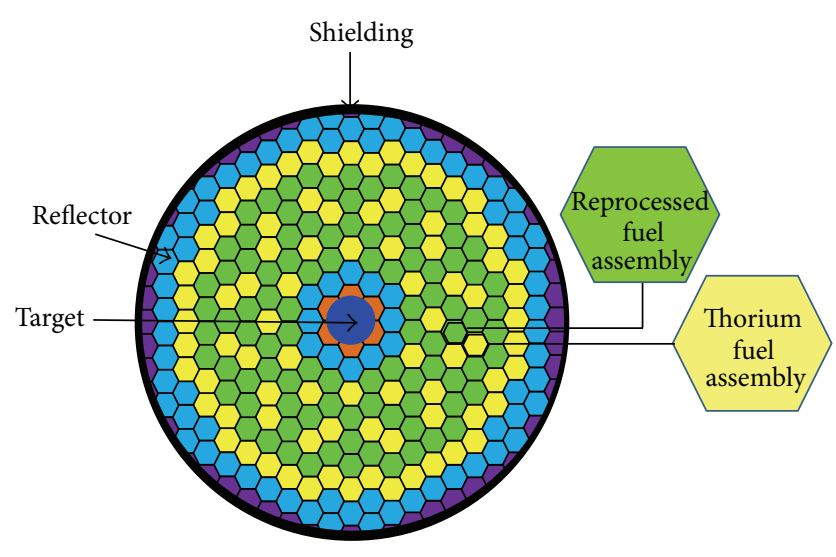

Figure 1: Seed and blanket ADS core layout.

of thorium fuel in blanket produces much less actinide compared with uranium fuel and the breeding ${ }^{233} \mathrm{U}$ from thorium helps to compensate the burnt TRUs in reprocessed fuel, thus reducing the reactivity swing. Besides, the power contribution from the thorium fuel assemblies increases due to burnup, thus flattening the power peaking factor over the core life time. The core layout is shown in Figure 1. This system is designed to obtain a fast neutron spectrum system since the fast spectra are found to be advantageous for actinide transport from the standpoint that actinide fissionto-capture ratios are high in them [6]. Neutron energy spectra for target and fuel regions in seed and blanket ADS core are shown in Figure 2.

\section{Methodology}

In order to investigate the influence of the ${ }^{232}$ Th capture cross section on reactivity calculation of the fast spectrum system, calculations of sensitivity coefficient of $k_{\text {eff }}$ of the seed and blanket ADS using thorium-reprocessed fuel for ${ }^{232}$ Th capture cross section of JENDL-4.0 and ENDF/B-VII [7] are conducted by using SAGEP code [8] with 107-group cross section data obtained by SRAC2006-CITATION [9]. SAGEP is the code which calculates sensitivity coefficients in a multidimensional system on the basis of generalized perturbation theory. The sensitivity calculation scheme is presented in Figure 3. The sensitivity coefficient gives the relative change in the system multiplication factor value as a function of the relative change in the ${ }^{232}$ Th capture cross section data [10].

$V$ shows the covariance matrix that expresses the correlation in the ${ }^{232} \mathrm{Th}(n, \gamma)$ cross section for energy groups. In this study, it is generated in a 107-group energy structure to map them into the same group structure as the sensitivity coefficients [10]. The covariance data for ${ }^{232}$ Th capture cross section is derived from the JENDL-4.0 and ENDF/B-VII evaluated nuclear data file using ERRORR module for NJOY99 code [11]. The uncertainty $(\delta E)$ of the $k_{\text {eff }}$ for ${ }^{232}$ Th capture cross section is derived as in (1) as follows:

$$
\delta E=\sqrt{S^{T} \cdot V \cdot S}
$$

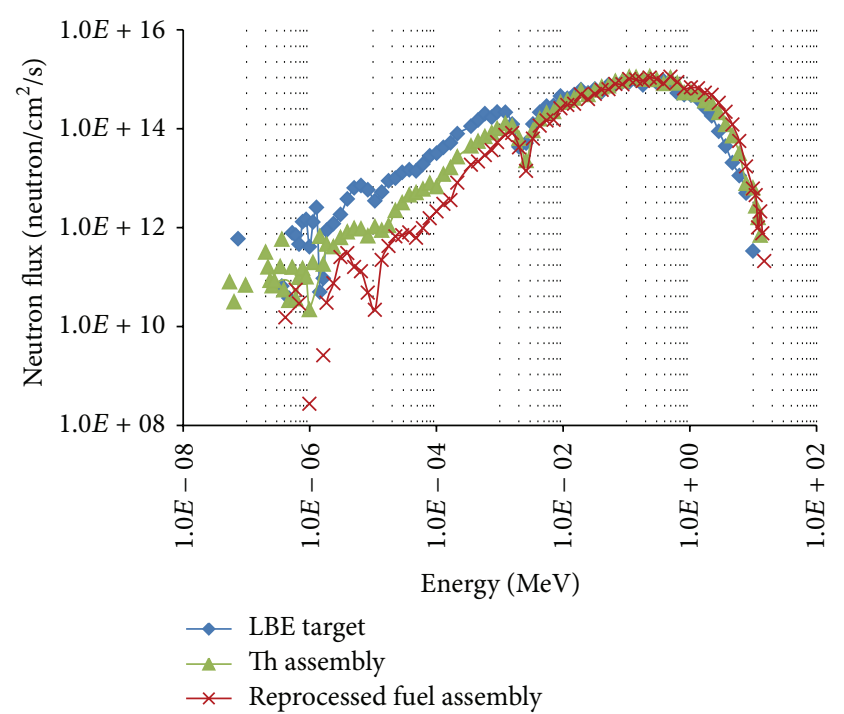

FIGURE 2: Neutron energy spectra for target and fuel regions in ADS core [3].

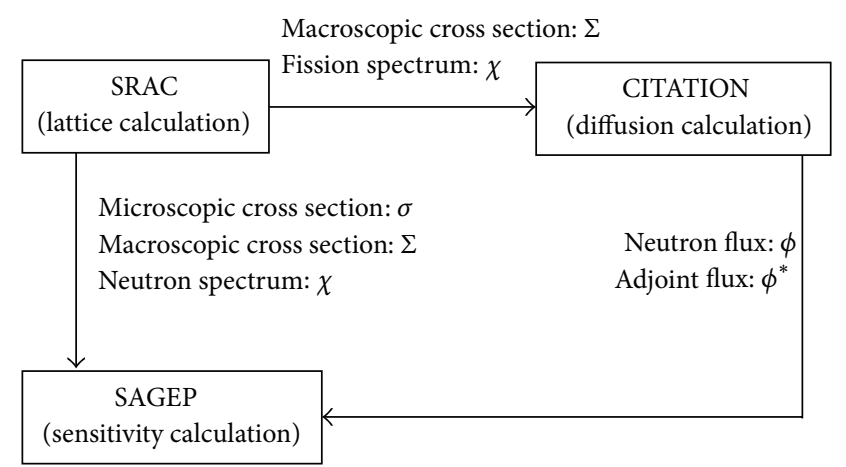

FIGURE 3: Sensitivity calculation scheme.

where $S$ is sensitivity coefficient of $k_{\text {eff }}$ in a 107-group energy structure obtained by SAGEP code and the superscript $T$ means transposition,

$$
S=\left[\begin{array}{c}
S_{1} \\
S_{2} \\
\vdots \\
S_{107}
\end{array}\right]
$$

and $V$ is the covariance matrix for $(n, \gamma)$ reaction of ${ }^{232} \mathrm{Th}$ in a 107-group energy structure obtained by NJOY99 code:

$$
V=\left[\begin{array}{cccc}
V_{1,1} & V_{1,2} & \cdots & V_{1,107} \\
V_{2,1} & V_{2,2} & \cdots & V_{2,107} \\
\vdots & \vdots & \ddots & \vdots \\
V_{107,1} & V_{107,2} & \cdots & V_{107,107}
\end{array}\right]
$$

\section{Results and Discussion}

Figure 4 illustrates the sensitivity coefficients of multiplication factor for capture reaction of ${ }^{232}$ Th of JENDL-4.0 and 


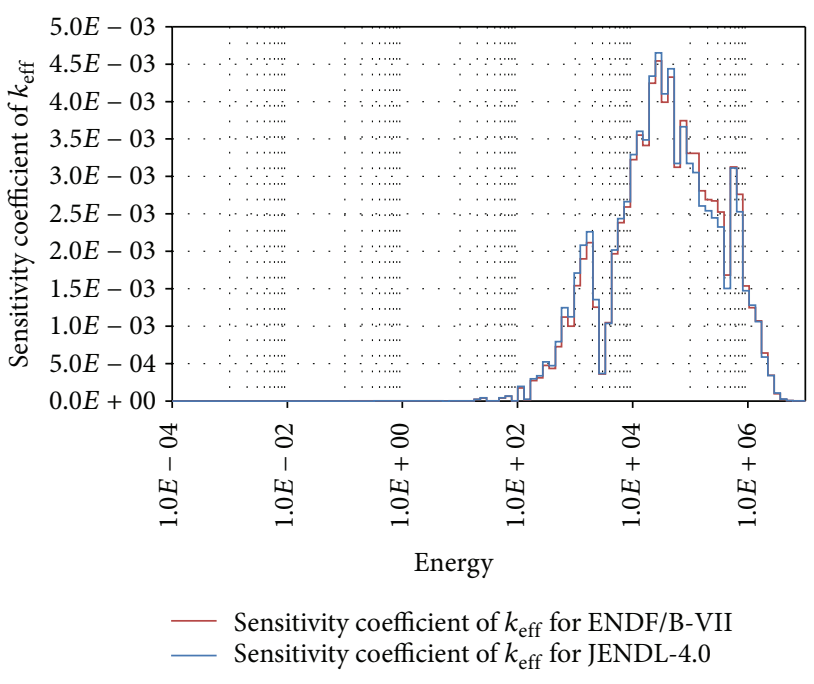

FIGURE 4: Sensitivity coefficient of $k_{\text {eff }}$ for ${ }^{232}$ Th capture cross section of JENDL-4.0 and ENDF/B-VII library.

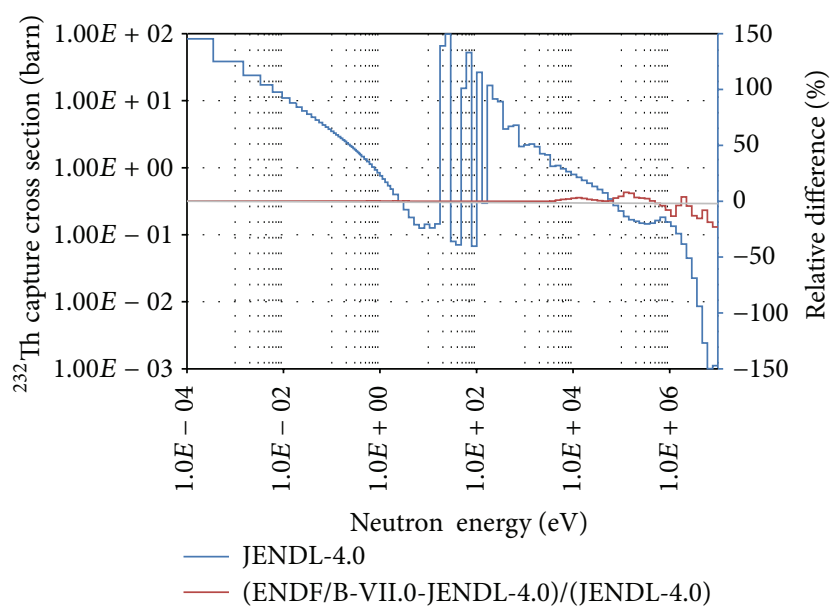

FIGURE 5: ${ }^{232}$ Th capture cross sections of JENDL-4.0 and relative difference among JENDL-4.0 and ENDF/B-VII.

ENDF/B-VII cross section library obtained using SAGEP code. As we can see from the figure, sensitivity coefficients of ${ }^{232}$ Th capture cross section of two libraries mainly concentrate on the fast energy range and are almost the same because the difference between two libraries is very small (Figure 5). The covariance data of two libraries was generated using NJOY99 code and illustrated in Figures 6(a) and 6(b). As we can see from Figures 6(a) and 6(b), the correlation in the $(n, \gamma)$ cross section for energy groups of JENDL-4.0 library is larger than that of ENDF/B-VII, especially in the energy range from $10 \mathrm{eV}$ to $0.5 \mathrm{MeV}$, and it is the source to cause the difference in the $k_{\text {eff }}$ uncertainty between two libraries. As derived from (1), the uncertainty of the $k_{\text {eff }}$ caused by ${ }^{232}$ Th capture cross section of JENDL-4.0 is about $1.3 \%$. This uncertainty would cause a significant influence in reactivity calculation in the thorium utilized ADS evaluation. Thus, the ${ }^{232}$ Th capture cross section of JENDL-4.0 is needed to be adjusted to give

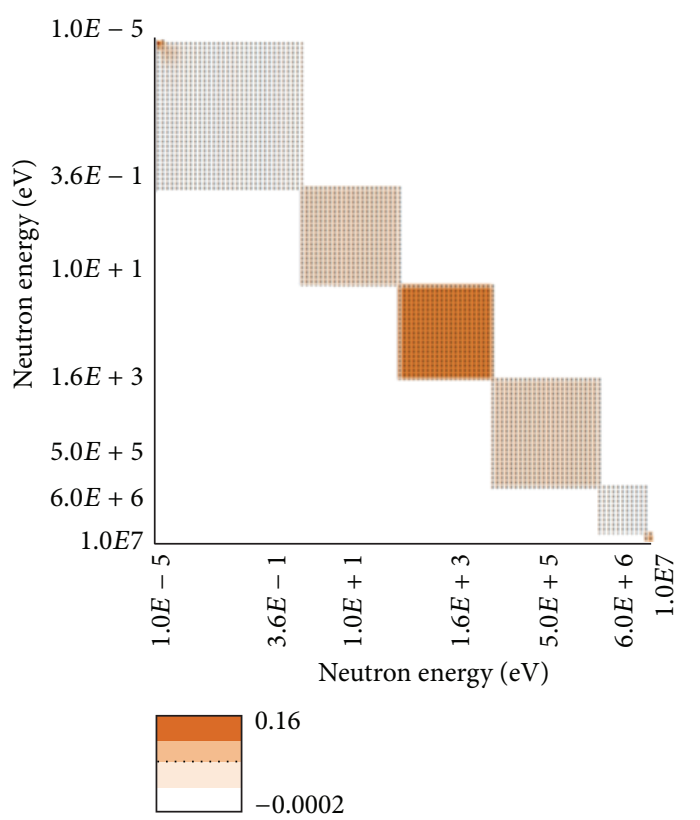

(a)

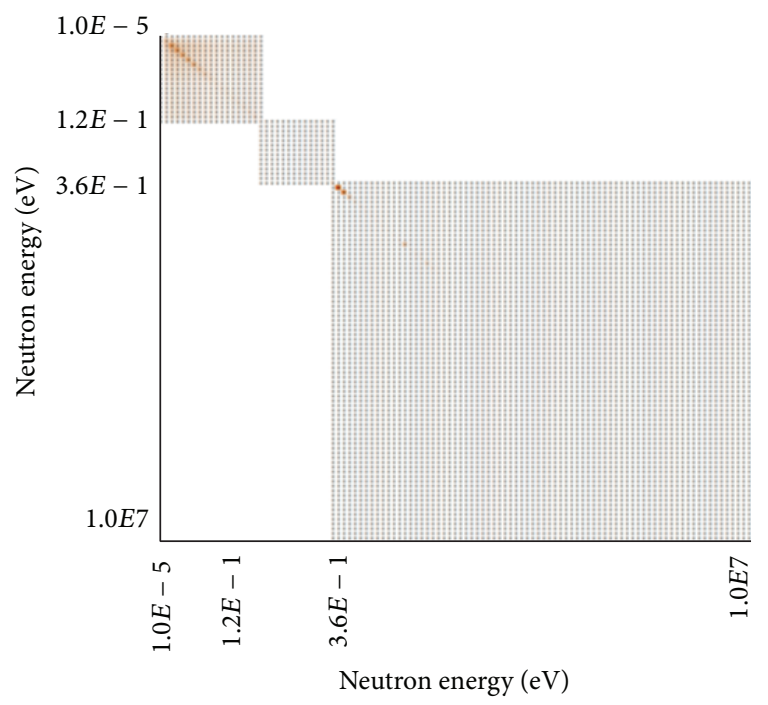

(b)

FIGURE 6: $(\mathrm{a}$ and $\mathrm{b}){ }^{232}$ Th covariance matrix of $(n, \gamma)$ reaction of JENDL-4.0 and ENDF/B-VII.

more reliable results on reactivity calculation for the fast system. On the other hand, the impact of uncertainty of ${ }^{232}$ Th capture cross section of ENDF/B-VII is small (0.1\%). Therefore, its influence on reactivity calculation of Th fuel utilized ADS is not significant. It can be used for the fast spectrum ADS design purpose.

\section{Conclusions}

The impact of thorium cross section uncertainty on thorium utilized ADS reactivity calculation is investigated in this study. The sensitivity calculations were done on ${ }^{232}$ Th capture 
cross section of JENDL-4.0 and ENDF/B-VII. As a result, the uncertainty of the $k_{\text {eff }}$ caused by ${ }^{232}$ Th capture cross section of JENDL-4.0 is about $1.3 \%$. The impact of uncertainty of ${ }^{232} \mathrm{Th}$ capture cross section of ENDF/B-VII is small $(0.1 \%)$. The strong correlation in the $(n, \gamma)$ cross section for energy group of JENDL-4.0 library is the origin of the difference in $k_{\text {eff }}$ uncertainty between JENDL-4.0 and ENDF/B-VII libraries. The uncertainty of JENDL-4.0 is needed to be enhanced in order to provide more reliable results on reactivity calculation for the fast system. The influence of thorium capture cross section on reactivity calculation of ADS system is not significant; thus, it can be used in the thorium utilized ADS design calculation.

\section{Conflict of Interests}

The authors declare that there is no conflict of interests regarding the publication of this paper.

\section{References}

[1] A. Herrera-Martinez, Transmutation of nuclear waste in accelerator-driven systems [Ph.D. thesis], University of Cambridge, Cambridge, UK, 2004.

[2] Ismail, P. H. Liem, N. Takaki, and H. Sekimoto, "Performance of natural uranium- and thorium-fueled fast breeder reactors (FBRs) for ${ }^{233} \mathrm{U}$ fissile production," Progress in Nuclear Energy, vol. 50, no. 2-6, pp. 290-294, 2008.

[3] T. M. Vu and T. Kitada, "Transmutation strategy using thoriumreprocessed fuel ADS for future reactors," Science and Technology of Nuclear Installations, vol. 2013, Article ID 674638, 5 pages, 2013.

[4] K. Shibata, "JENDL-4.0: a new library for nuclear science and engineering," Journal of Nuclear Science and Technology, vol. 48, pp. 1-30, 2011.

[5] T. M. Vu, T. Fujii, K. Wada et al., "Accuracy of thorium cross section of JENDL-4.0 library in thorium based fuel core evaluation," Annals of Nuclear Energy, vol. 57, pp. 173-178, 2013.

[6] H. R. Trellue, Reduction of the radiotoxicity of spent nuclear fuel using a two-tiered system comprising light water reactors and accelerator-driven systems [Ph.D. thesis], University of New Mexico, 2003.

[7] M. B. Chadwick, P. Obložinský, M. Herman et al., "ENDF/BVII.0: Next generation evaluated nuclear data library for nuclear science and technology," Nuclear Data Sheets, vol. 107, no. 12, pp. 2931-3060, 2006.

[8] A. Hara, T. Takeda, and Y. Kikuchi, "SAGEP: two-dimensional sensitivity analysis code based on generalized pertubation theory," Tech. Rep. JAERI-M 84-027, JAERI, 1984.

[9] K. Okumura, T. Kugo, K. Kaneko, and K. Tsuchihashi, "SRAC2006: a comprehensive neutronics calculation code system," Tech. Rep. JAEA-Data/Code 2007-004, JAEA, 2007.

[10] T. Ivanova, I. Duhamel, and E. Letang, "Impact of cross section covariance data on results of high-confidence criticality validation," Journal of the Korean Physical Society, vol. 59, no. 2, pp. 1170-1173, 2011.

[11] Los Alamos National Laboratory, NJOY99.0, Code System for Producing Pointwise and Multigroup Neutron and Photon Cross Sections from ENDF/B Data, Los Alamos National Laboratory, Los Alamos, NM, USA, 2000. 


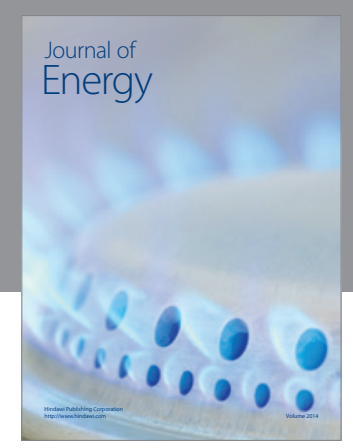

Journal of

Industrial Engineering
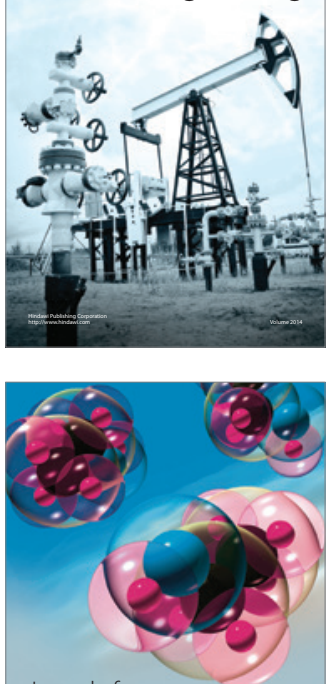

Fuels
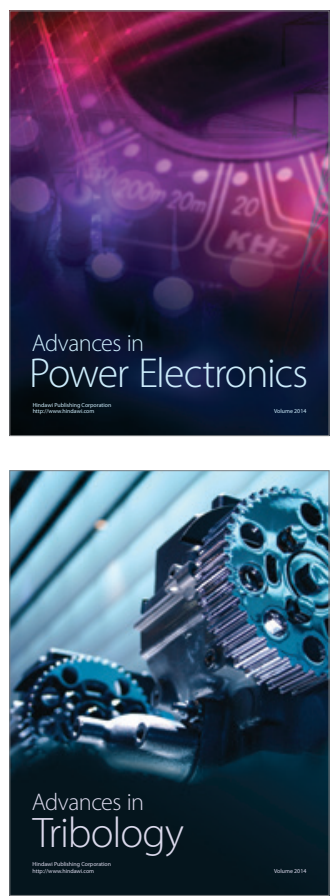

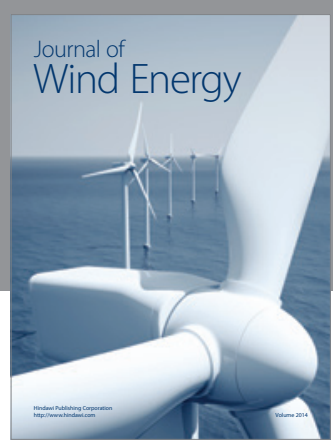

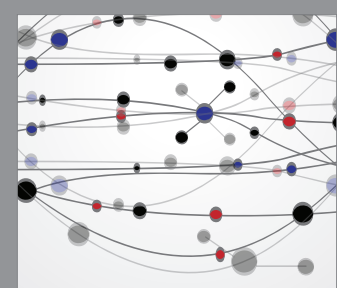

The Scientific World Journal

Submit your manuscripts at http://www.hindawi.com

Journal of

Structures
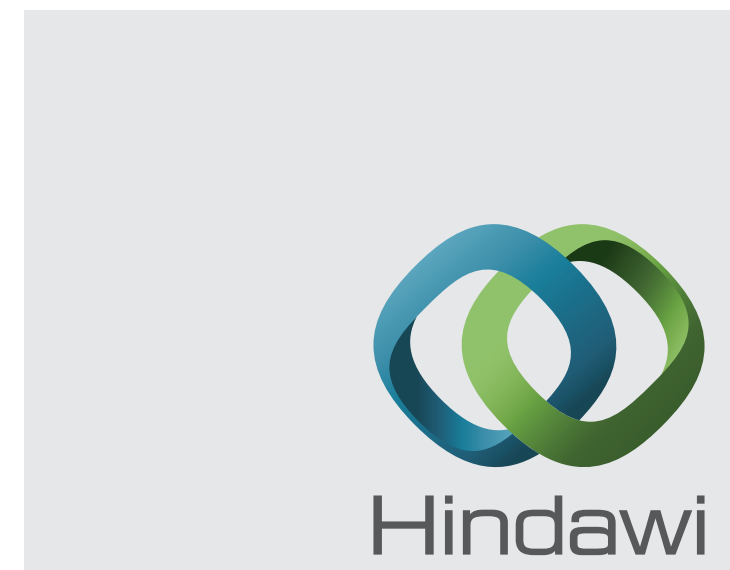

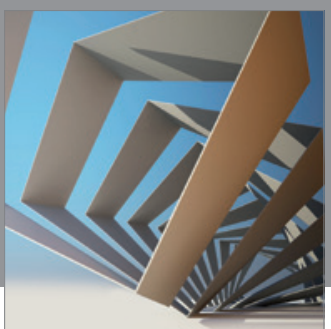

Rotating

Machinery
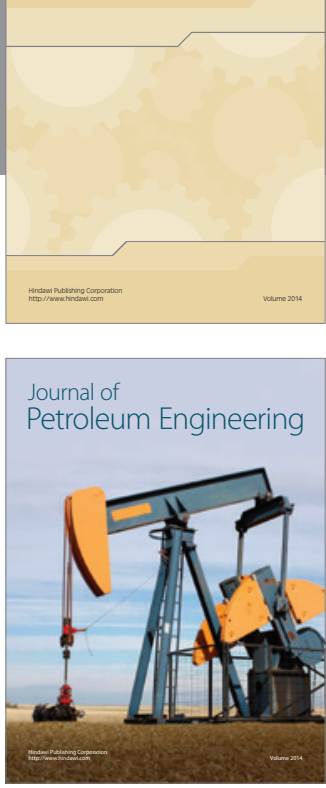

Journal of

Solar Energy
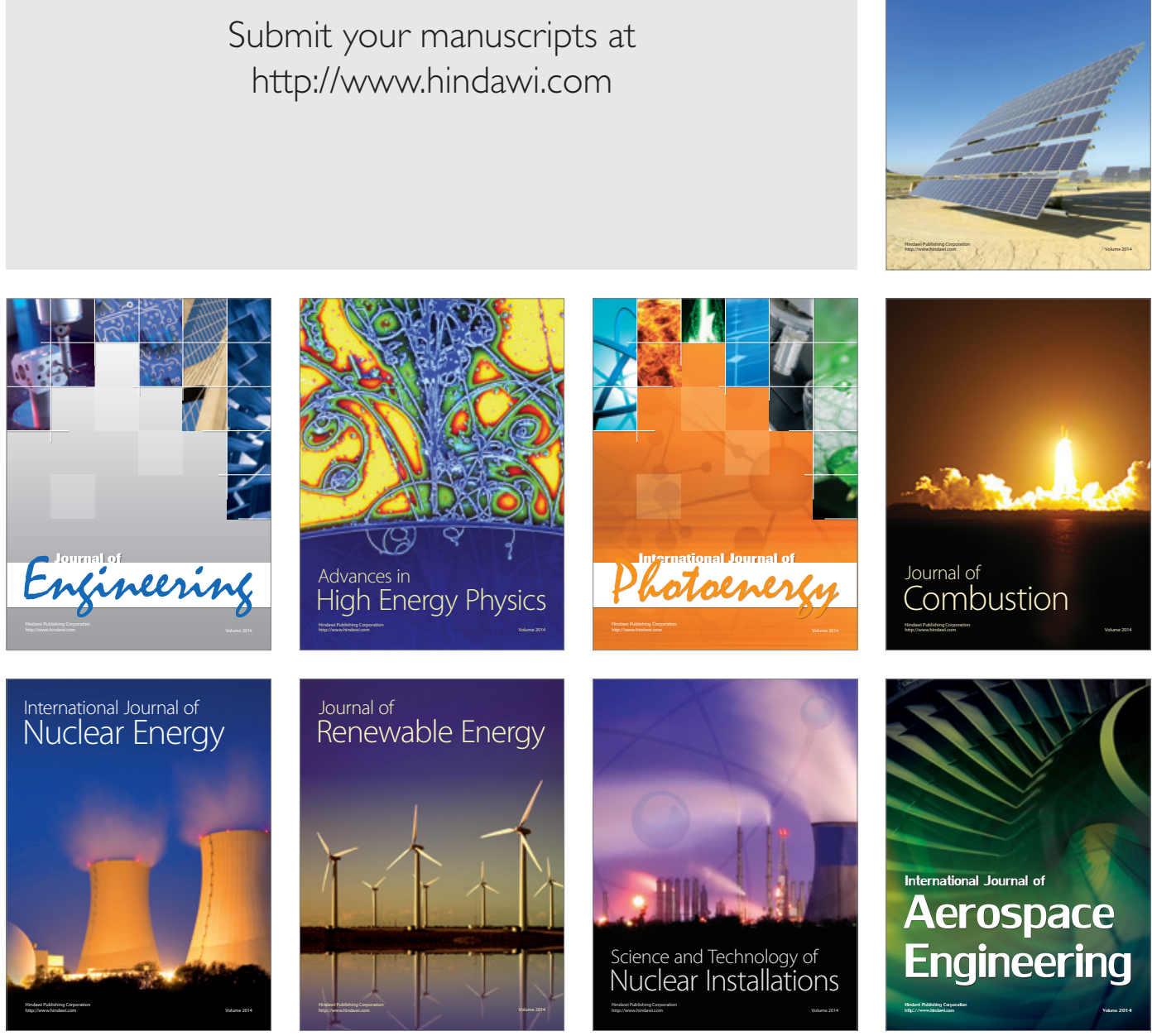